\title{
Li-Fi (Light Fidelity)-The Future Technology in Wireless Communication
}

\author{
K. Harshitha, A. Chaithra, N.A. Poojitha and B. Raghavendra Rao
}

\begin{abstract}
Whether you're using wireless internet in a coffee shop, stealing it from the guy next door, or competing for bandwidth at a conference, you have probably gotten frustrated at the slow speeds you face when more than one device is tapped into the network. As more and more people and their many devices access wireless internet, clogged airwaves are going to make it. One germen phycist. Harald Haas has come up with a solution he calls "data through illumination" -taking the fibber out of fiber optic by sending data through an ILED light bulb that varies in intensity faster than the human eye can follow. It's the same idea band behind infrared remote controls but far more powerful. Haas says his invention, which he calls DLIGHT, can produce data rates faster than 10 megabits per second, which is speedier than your average broadband connection. He envisions a future where data for laptops, smart phones, and tablets is transmitted through the light in a room. And security would be snap - if you can't see the light, you can't access the data.
\end{abstract}

Keywords--- LED (Light emitted diode), Wi-Fi, VLC.

\section{INTRODUCTION}

L IFI is transmission of data through illumination by taking the fiber out of fiber optics by sending data through a ${ }^{2}$ LED light bulb that varies in intensity faster than the human eye can follow. $\mathrm{Li}-\mathrm{Fi}$ is the term some have used to label the fast and cheap wireless-communication system, which is the optical version of Wi-Fi. The term was first used in this context by Harald Haas in his TED Global talk on Visible Light Communication. "At the heart of this technology is a new generation of high brightness light-emitting diodes", says Harald Haas from the University of Edinburgh, UK, "Very simply, if the LED is on, you transmit a digital 1, if it's off you transmit a 0 ,"Haas says, "They can be switched on and off very quickly, which gives nice opportunities for transmitted data."It is possible to encode data in the light by varying the rate at which the LEDs flicker on and off to give different strings of $1 \mathrm{~s}$ and $0 \mathrm{~s}$. The LED intensity is modulated so rapidly that human eye cannot notice, so the output appears constant. More sophisticated techniques could dramatically increase VLC data rate. Terms at the University of Oxford and the

\footnotetext{
K. Harshitha, Dept of CSE, SSEC, Bangalore, India. E-mail:harshigowda97@gmail.com

A. Chaithra Dept of CSE, SSEC, Bangalore, India. E-mail:chaithraarunachalam@gmail.com

N.A. Poojitha Dept of CSE, SSEC, Bangalore, India. E-mail:Poojitharoy143@gmail.com

B. Raghavendra Rao, Assistant Professor, Dept of CSE, SSEC, Bangalore.E-mail:raghavendrarao.cse@sairamce.edu.in DOI:10.9756/BIJSESC.8241
}

University of Edingburgh are focusing on parallel data transmission using array of LEDs, where each LED transmits a different data stream. Other group are using mixtures of red, green and blue LEDs to alter the light frequency encoding a different data channel. $\mathrm{Li}-\mathrm{Fi}$, as it has been dubbed, has already achieved blisteringly high speed in the lab. Researchers at the Heinrich Hertz Institute in Berlin, Germany, have reached data rates of over 500 megabytes per second using a standard white-light LED. The technology was demonstrated at the 2012 Consumer Electronics Show in Las Vegas using a pair of Casio smart phones to exchange data using light of varying intensity given off from their screens, detectable at a distance of up to ten metres.

In October 2011 a number of companies and industry groups formed the Li-Fi Consortium, to promote high-speed optical wireless systems and to overcome the limited amount of radio based wireless spectrum available by exploiting a completely different part of the electromagnetic spectrum. The consortium believes it is possible to achieve more than 10 Gbps, theoretically allowing a high-definition film to be downloaded in 30 seconds.

\section{WORKING TECHNOLOGY}

The LEDs can be switched on and off very quickly, which gives nice opportunities for transmitting data." So what you require at all are some LEDs and a controller that code data into those LEDs. We have to just vary the rate at which the LED's flicker depending upon the data we want to encode. Further enhancements can be made in this method, like using an array of LEDs for parallel data transmission, or using mixtures of red, green and blue LEDs to alter the light's frequency with each frequency encoding a different data channel. Such advancements promise a theoretical speed of 10 Gbps - meaning you can download a full high-definition film in just 30 seconds. Simply awesome! But blazingly fast data rates and depleting bandwidths worldwide are not the only reasons that give this technology an upper hand. Since Li-Fi uses just the light, it can be used safely in aircrafts and hospitals that are prone to interference from radio waves. This can even work under water where Wi-Fi fails completely, thereby throwing open endless opportunities for military operations.

Imagine only needing to hover under a street lamp to get public internet access, or downloading a movie from the lamp on your desk. There's a new technology on the block which could, quite literally as well as metaphorically, 'throw light on' how to meet the ever-increasing demand for high-speed wireless connectivity. Radio waves are replaced by light waves in a new method of data transmission which is being called Li-Fi. Light-emitting diodes can be switched on and off 
faster than the human eye can detect, causing the light source to appear to be on continuously.

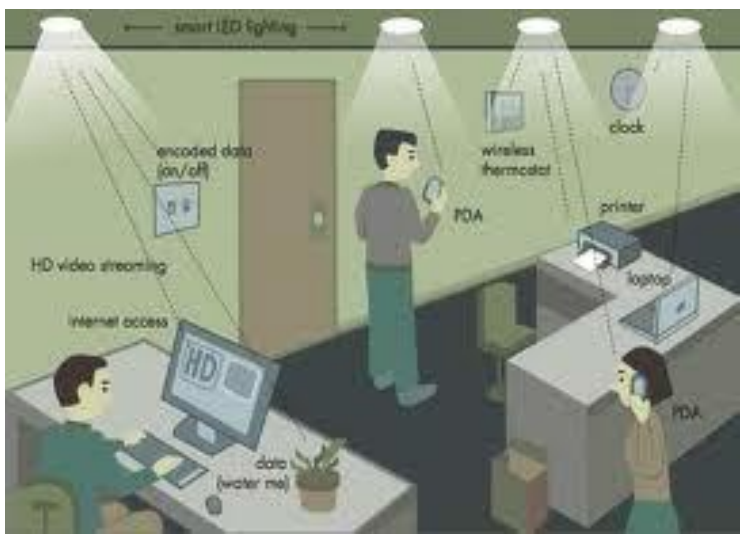

Figure 1: Li-Fi Enviorment

A flickering light can be incredibly annoying, but has turned out to have its upside, being precisely what makes it possible to use light for wireless data transmission. Lightemitting diodes (commonly referred to as LEDs and found in traffic and street lights, car brake lights, remote control units and countless other applications) can be switched on and off faster than the human eye can detect, causing the light source to appear to be on continuously, even though it is in fact 'flickering'. This invisible on-off activity enables a kind of data transmission using binary codes: switching on an LED is a logical ' 1 ', switching it off is a logical ' 0 '. Information can therefore be encoded in the light by varying the rate at which the LEDs flicker on and off to give different strings of $1 \mathrm{~s}$ and 0s. This method of using rapid pulses of light to transmit information wirelessly is technically referred to as Visible Light Communication (VLC), though it's potential to compete with conventional $\mathrm{Wi}-\mathrm{Fi}$ has inspired the popular characterization Li-Fi.

\section{A. Visible Light Communication (VLC) \\ "A potential solution to the global wireless spectrum shortage"}

LiFi (Light Fidelity) is a fast and cheap optical version of $\mathrm{Wi}$ - Fi, the technology of which is based on Visible Light Communication (VLC). ${ }^{4}$ VLC is a data communication medium, which uses visible light between $400 \mathrm{THz}(780 \mathrm{~nm})$ and $800 \mathrm{THz}(375 \mathrm{~nm})$ as optical carrier for data transmission and illumination. It uses fast pulses of light to transmit information wirelessly.

The main components of this communication system are

1. A high brightness white LED, Which acts as a communication source and

2. A silicon photodiode which shows good response to visible wavelength region serving as the receiving element? ${ }^{3}$ LED can be switched on and off to generate digital strings of $1 \mathrm{~s}$ and $0 \mathrm{~s}$. Data can be encoded in the light to generate a new data stream by varying the flickering rate of the LED. To be clearer, by modulating the LED light with the data signal, the LED illumination can be used as a communication source. As the flickering rate is so fast, the LED output appears constant to the human eye. A data rate of greater than $100 \mathrm{Mbps}$ is possible by using high speed LEDs with appropriate multiplexing techniques. VLC data rate can be increased by parallel data transmission using LED arrays where each LED transmits a different data stream. There are reasons to prefer LED as the light source in VLC while a lot of other illumination devices like fluorescent lamp, incandescent bulb etc. are available.

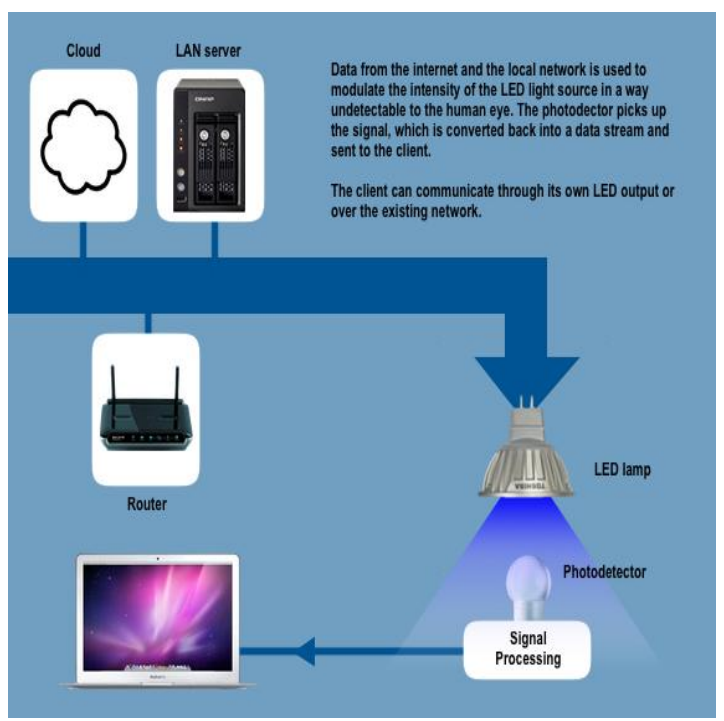

Figure 2: Data transmission using LED

\section{COMPARISON BETWEEN LI-FI \& WI-FI}

LI-FI is a term of one used to describe visible light communication technology applied to high speed wireless communication. It acquired this name due to the similarity to WI-FI, only using light instead of radio. ${ }^{5} \mathrm{WI}-\mathrm{FI}$ is great for general wireless coverage within buildings, and ${ }^{5}$ li-fi is ideal for high density wireless data coverage in confined area and for relieving radio interference issues, so the two technologies can be considered complimentary.

Table 1: Comparison Between Current and Future Wireless Technology

\begin{tabular}{|c|c|c|c|}
\hline S.No. & Parameter & Li-fi & Wi-fi \\
\hline 1. & Speed & $>1 \mathrm{~GB} / \mathrm{s}$ & Around $150 \mathrm{mb} / \mathrm{s}$ \\
\hline 2. & Medium of data transfer & Use light as carrier & $\begin{array}{c}\text { Use radio } \\
\text { spectrum }\end{array}$ \\
\hline 3. & Spectrum range & $\begin{array}{c}\text { Visible light has } \\
10000 \text { times more }\end{array}$ & $\begin{array}{c}\text { Having less } \\
\text { spectrum range } \\
\text { than VLC }\end{array}$ \\
\hline 4. & Cost & Cheaper & Expensive \\
\hline 5. & Network topology & Point-to-Point & Point-to-Point \\
\hline 6. & Operating Frequency & $\begin{array}{c}\text { Hundreds of Tera } \\
\text { Hz }\end{array}$ & $2.4 \mathrm{GHz}$ \\
\hline
\end{tabular}

the current wireless technologies that can be used for transferring data between devices today, i.e. Wi-Fi, Bluetooth and IrDA. Only Wi-Fi currently offers very high data rates. 
The IEEE 802.11.n in most implementations provides up to $150 \mathrm{Mbit} / \mathrm{s}$ (in theory the standard can go to $600 \mathrm{Mbit} / \mathrm{s}$ ) although in practice you receive considerably less than this. Note that one out of three of these is an optical technology.

\section{A. How Li Fi it is Different?}

Li-Fi technology is based on LEDs for the transfer of data. The transfer of the data can be with the help of all kinds of light, no matter the part of the spectrum that they belong. That is, the light can belong to the invisible, ultraviolet or the visible part of the spectrum. Also, the speed of the internet is incredibly high and you can download movies, games, music etc in just a few minutes with the help of this technology. Also, the technology removes limitations that have been put on the user by the Wi-Fi. You no need to be in a region that is Wi-Fi enabled to have access to the internet. You can simply stand under any form of light and surf the internet as the connection is made in case of any light presence. There cannot be anything better than this technology.

\section{APPLICATION OF ${ }^{4} \mathrm{LI}-\mathrm{FI}$}

\section{A. Airlines}

Airline Wi-Fi. Ugh. Nothing says captive audience like having to pay for the "service" of dial-up speed Wi-Fi on the plane. And don't get me started on the pricing. The best I've heard so far is that passengers will "soon" be offered a "highspeed like" connection on some airlines. United is planning on speeds as high as 9.8 Mbps per plane. Uh, I have twice that capacity in my living room. And at the same price as checking a bag, I expect it. Li-Fi could easily introduce that sort of speed to each seat's reading light. Its better than listening to you tell me about your wildly successful son, ma'am.

\section{B. Smarter Power Plants}

Wi-Fi and many other radiation types are bad for sensitive areas. Like those surrounding power plants. But power plants need fast, inter-connected data systems to monitor things like demand, grid integrity and (in nuclear plants) core temperature. The savings from proper monitoring at a single power plant can add up to hundreds of thousands of dollars. Li-Fi could offer safe, abundant connectivity for all areas of these sensitive locations. Not only would this save money related to currently implemented solutions, but the draw on a power plant's own reserves could be lessened if they haven't yet converted to LED lighting

\section{Undersea Awesomeness}

Underwater ROVs, those favourite toys of treasure seekers and James Cameron, operate from large cables that supply their power and allow them to receive signals from their pilots above. ROVs work great, except when the tether isn't long enough to explore an area, or when it gets stuck on something. If their wires were cut and replaced with light - say from a submerged, high-powered lamp - then they would be much freer to explore. They could also use their headlamps to communicate with each other, processing data autonomously and referring findings periodically back to the surface, all the while obtaining their next batch of orders.
Say there's an earthquake in New York. Or a hurricane. Take your pick - it's a wacky city. The average New Yorker may not know what the protocols are for those kinds of disasters. Until they pass under a street light, that is. Remember, with Li-Fi, if there's light, you're online. Subway stations and tunnels, common dead zones for most emergency communications, pose no obstruction. Plus, in times less stressing cities could opt to provide cheap high-speed Web access to every street corner.

\section{USES IN VARIOUS AREAS}

Can be used in the places where it is difficult to lay the optical fiber like hospitals. In operation theatre LiFi can be used for modern medical instruments. In traffic signals LiFi can be used which will communicate with the LED lights of the cars and accident numbers can be decreased. Thousand and millions of street lamps can be transferred to LiFi lamps to transfer data. In aircraft $\mathrm{LiFi}$ can be used for data transmission. It can be used in petroleum or chemical plants where other transmission or frequencies could be hazardous.

\section{CONCLUSION}

The possibilities are numerous and can be explored further. If his technology can be put into practical use, every bulb can be used something like a Wi-Fi hotspot to transmit wireless data and we will proceed toward the cleaner, greener, safer and brighter future. The concept of Li-Fi is currently attracting a great deal of interest, not least because it may offer a genuine and very efficient alternative to radio-based wireless. As a growing number of people and their many devices access wireless internet, the airwaves are becoming increasingly clogged, making it more and more difficult to get a reliable, high-speed signal. This may solve issues such as the shortage of radio-frequency bandwidth and also allow internet where traditional radio based wireless isn't allowed such as aircraft or hospitals. One of the shortcomings however is that it only work in direct line of sight.

\section{REFERENCES}

[1] seminarprojects.com/s/seminar-report-on-lifi

[2] http://en.wikipedia.org/wiki/Li-Fi

[3] http://teleinfobd.blogspot.in/2012/01/what-is-lifi.html

[4] Technopits.blogspot.comtechnology.cgap.org/2012/01/11/a-lifi-world/

[5] Will Li-Fi be the new Wi-Fi?, New Scientist, by Jamie Condliffe, dated 